\title{
Effect of Backscattered Radiation on X-Ray Image Contrast
}

\author{
A. T. Naji ${ }^{1}$, M. S. Jaafar ${ }^{1}$, E. A. Ali ${ }^{2} \&$ S. K. J. Al-Ani ${ }^{3}$ \\ ${ }^{1}$ School of Physics, University of Science Malaysia (USM), Pulau Pinang, Malaysia \\ ${ }^{2}$ College of Engineering, University of Science and Technology, Sana'a, Yemen \\ ${ }^{3}$ Department of Physics, College of Science, University of Baghdad, Baghdad, Iraq \\ Correspondence: S. K. J. Al-Ani, Department of Physics, College of Science, University of Baghdad, Baghdad, \\ Iraq. E-mail: salwan_kamal@yahoo.com
}

Received: October 27, 2016

Accepted: November 17, 2016 Online Published: January 31, 2017

doi:10.5539/apr.v9n1p105

URL: http://dx.doi.org/10.5539/apr.v9n1p105

\begin{abstract}
This paper assesses the effect of backscattered radiation on X-ray image contrast by evaluating the effect of backscatter reduction on X-ray image contrast. Contrast test tool RMI Densitometer, and different types of fabricated anti backscattered grids have been utilized in this study. The measurements are recorded at different exposure parameters such as X-ray tube peak voltage $(\mathrm{kVp})$, and X-rays intensities (mAs). For each exposure, the contrast of the image is evaluated by measuring the variation in optical densities for aluminium steps wedge. The results showed that the $\mathrm{x}$-ray image contrast can be enhanced by decreasing the amount of backscattered radiation, also the fabricated anti backscattered grids have a remarkable effect in the improvement of X-ray image contrast according to grid's capability in reducing backscattered radiation. In addition, the effectiveness of fabricated grids in improving image contrast depends on the grid's material and the geometrical design, as well as the radiation exposure parameters. The image contrast enhancements increased up to $36 \%$ with the use of crossed iron steel grid which placed under the film screen combination during exposure.
\end{abstract}

Keywords: contrast, anti-backscattered grid, x-ray, image quality

\section{Introduction}

The contrast of the image is one of the most important properties of X-ray image quality. Because of the X-ray film is a negative recorder and the degree of darkness of the film is quantified by the optical density (OD), the contrast could be expressed by the degree of difference in optical density between adjacent areas of the X-ray image. (Mohanta \& Khanaa, 2013, Bushberg, 2002)

The X-ray image contrast is a combination of subject contrast which refers to the contrast produced due to the anatomical structures under examination, and the receptor contrast which refers to the contrast produced as a result of the X-ray image receptor being employed (Bushong,2013).

To detect diagnostic radiograph details, a lesion must transmit a different X-ray beam intensity compared with the surrounding normal tissues. The difference in X-ray beam intensity through a lesion relative to the normal background is called subject contrast. The appearance of this subject contrast in the resultant radiograph is called image contrast (Huda \& Abrahams, 2015).

In a high contrast radiograph, individual objects stand out and their edges are clearly defined (high sharpness). Whereas, for low radiograph contrast individual objects loss some of their clarity while their edges become soft or blurred (Carver et al., 2012; Suetens, 2009).

With scattered photons, the contrast decreases and the edges of the profile become softened. The ideal radiograph is degraded in sharpness by scattered photons. This is because the scattered photons of the transmitted radiation do not possess the simple straight-line relationship between X-ray tube focus, patient point and image receptor. Hence, scattered photons modify the resulting intensity at the particular point in the image as it is shown in Figure 1 (Guy \& Flytche, 2005). 


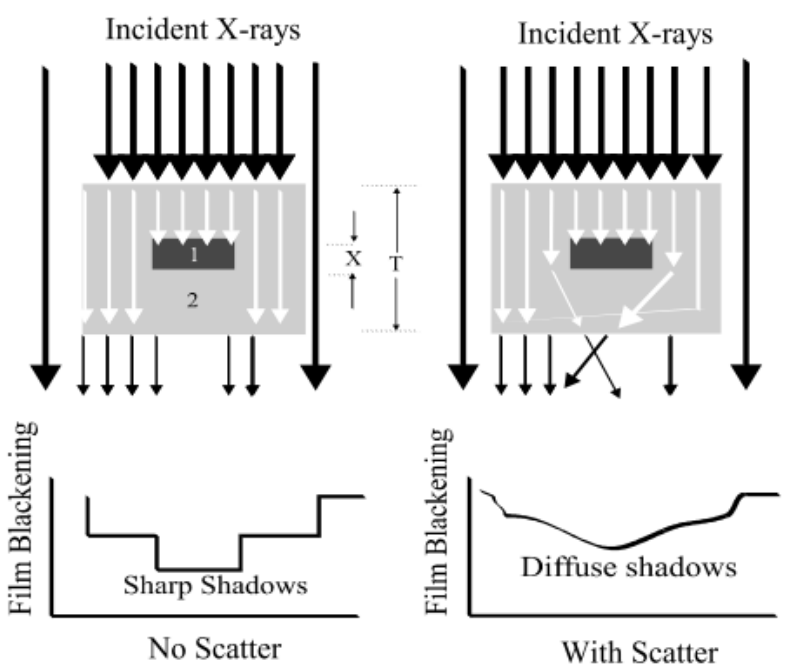

Figure 1. Model defines the X-ray image contrast (Guy \& Flytche, 2005)

According to Figure 1, the intensity at the middle of target object to be $\mathrm{I}_{1}$ and a reference point in the image just outside the object in the background to be $\mathrm{I}_{2}$ (Guy \& Flytche, 2005). Then the contrast, $\mathrm{C}$, is defined to be

$$
\mathrm{C}=\frac{\mathrm{I}_{1}-\mathrm{I}_{2}}{\mathrm{I}_{2}}
$$

For each of the intensities, the primary X-rays, $\mathrm{P}$ and the scattered X-rays, $\mathrm{S}$, that reach the detector as follows:

With assumption:

$$
\begin{aligned}
& \mathrm{I}_{1}=\mathrm{P}_{1}+\mathrm{S}_{1} \\
& \mathrm{I}_{2}=\mathrm{P}_{2}+\mathrm{S}_{2}
\end{aligned}
$$

$$
\mathrm{S}_{1} \cong \mathrm{S}_{2} \cong \mathrm{S}
$$

From Equations 1, 2, 3, and 4 the contrast cab be expressed as the following

$$
\mathrm{C}=\frac{\mathrm{P}_{1}-\mathrm{P}_{2}}{\mathrm{P}_{2}+\mathrm{S}}
$$

Equation 5 shows the effect of scattered radiation existence on reducing the contrast of resultant X-ray image.

It is assumed that the scatter contribution is the same to both regions (imaged target and its background). Therefore, Scatter radiation can greatly reduce X-ray image contrast to the extent that subtle opacities may not be visualized (Yumpu, 2015; Guy \& Flytche, 2005). Naji et al. (2016) introduced a method to reduce backscattered radiation which can affect on image contrast by using iron steel grid with several metal plates

Contrast enhancement is an image optimization technique that refers to increasing the intensity difference between the region of interest and background (Godwin et al., 2014). Image contrast enhancement is an important aspect in medical applications due to the fact that visual examination of medical images is essential in the diagnosis of many diseases (Mohanta \& Khanaa, 2013).

This study is performed to investigate a method that reduces the backscattered photons which affect the X-ray image contrast. Although lead and iron have best attenuating values for incident X-ray at radiographic energy range, iron and aluminium exhibited the least amount of backscattered radiation dose, and thus they can be useful materials in designing anti-scattered X-ray grid and lining the walls of $\mathrm{x}$-ray room or the back panel of radiographic film cassette. Therefore, the X-ray image's contrast is evaluated by using several anti-backscattered grids namely: crossed iron, linear iron, crossed aluminium and linear aluminium which proved a high capability in attenuating the backscattered X-ray up to $49 \%$ (Naji, 2016). These grids may be used to enhance the X-ray image contrast as well as to improve the radiation protection inside the X-ray room. 


\section{Experiments}

\subsection{Equipment}

A number of equipment are utilized in this study which included X-ray machines, contrast test tool (RMI aluminium stepwedge, SN: 117-1189), RMI Densitometer (Gamex), radiographic image Autoprocessor (Minolta SRX-101A). Some anti-backscattered grids are fabricated in this study in order to reduce backscattered radiation which in turn affects the X-ray image contrast. These grids constructed from various material (iron steel and aluminium) with different geometric designs (crossed and linear), the shown in Figure 2.

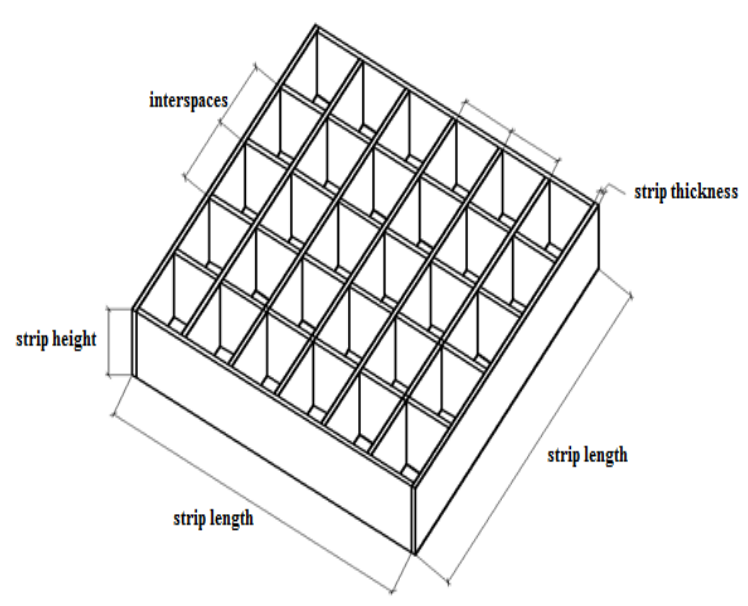

The schematic design of crossed grids

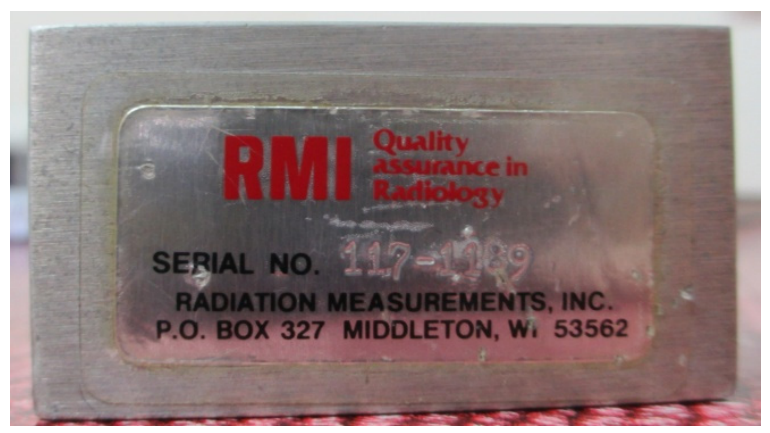

RMI Aluminium step wedge

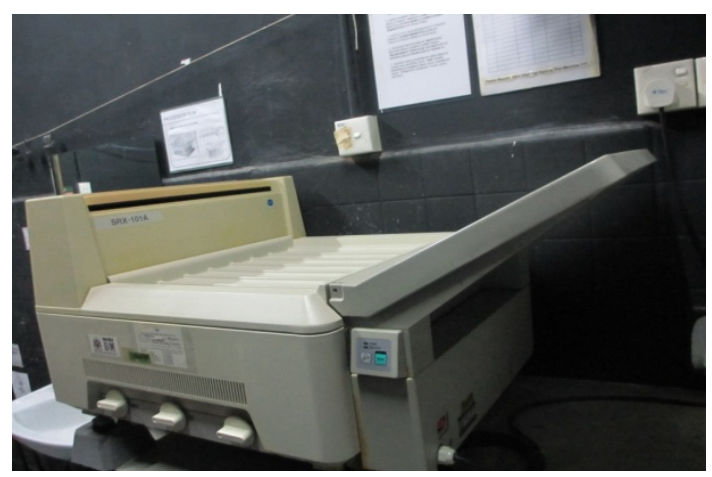

X-ray Image processor (SRX-101A)

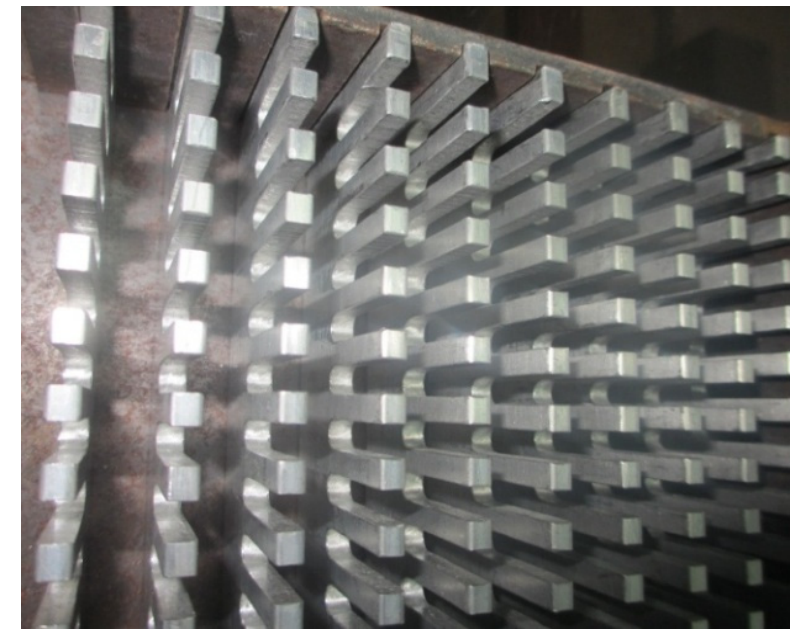

Anti-backscattered radiation grid (linear)

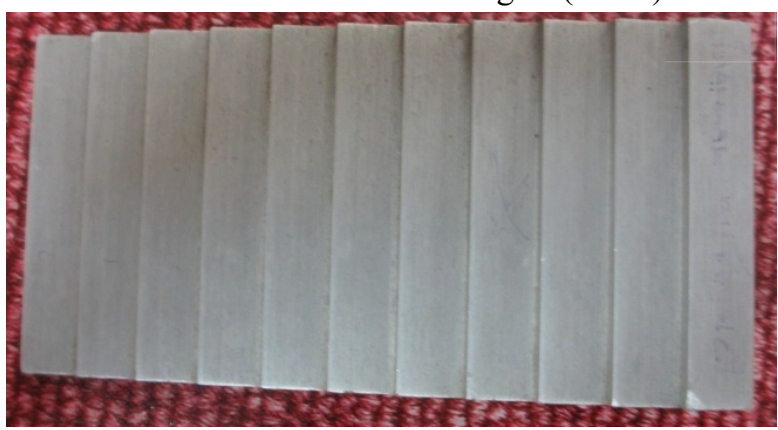

RMI Aluminium step wedge



RMI Densitometer

Figure 2. Experimental equipment 


\subsection{Procedures of Measuring Image's Contrast}

Contrast test tool and Densitometer are adopted to estimate X-ray image's contrast. For each exposure, the contrast test tool is placed on the film cassette and the collimator is adjusted to cover the test tool accurately. Various tube peak voltages $(\mathrm{kVp})$ and $\mathrm{X}$-rays intensity (mAs) are performed to produce X-ray images. The differences in step wedge thicknesses give rise to differences in optical density.

After developing the films, the variation of optical density for steps wedge are estimated by densitometers which in turn can be utilized in the assessment of image's contrast. The effect of utilizing anti-backscatter grids on image's contrast was evaluated by comparing image's contrast with and without using anti-backscatter grids which were positioned under film screen combination during image acquisition, as shown in Figure 3,

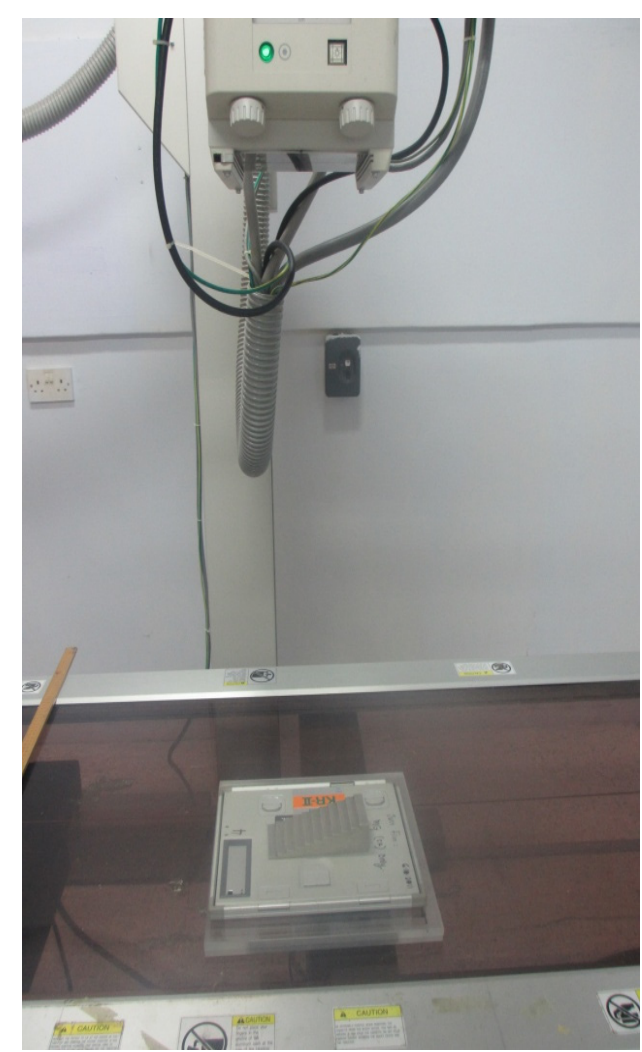

(a)

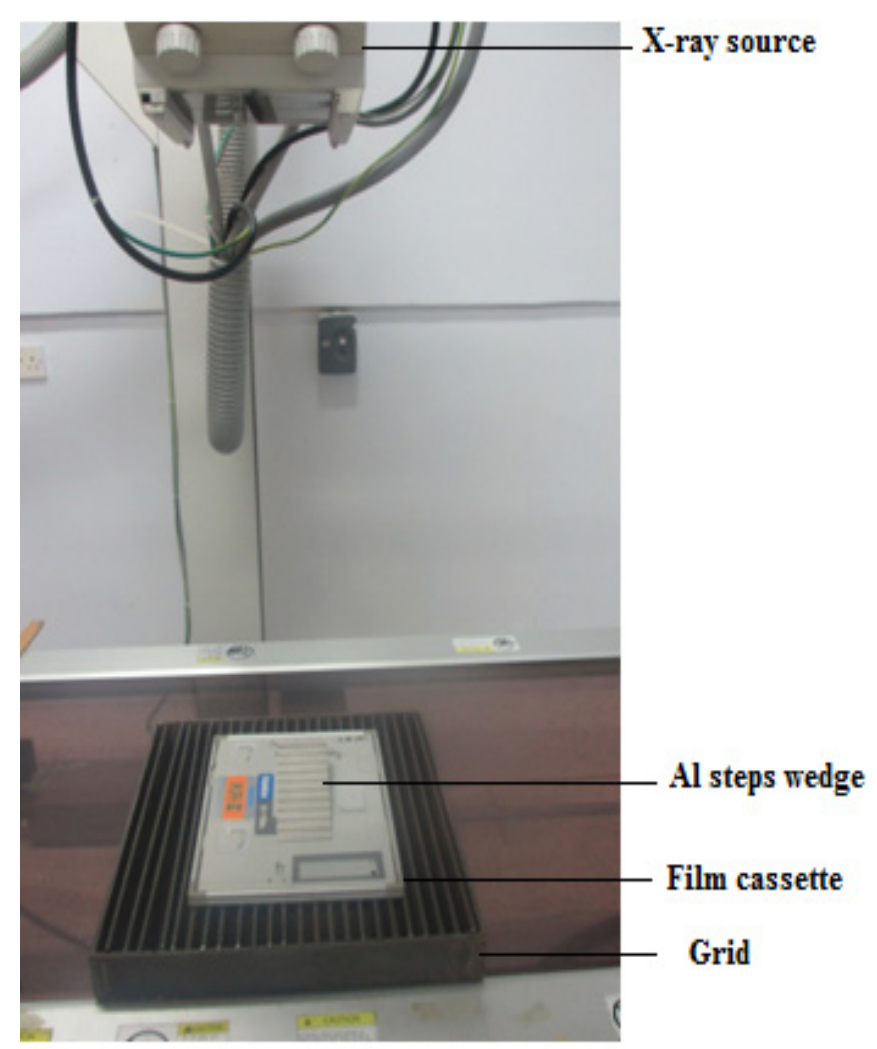

(b)

Figure 3. Evaluation of image's contrast. (a) Imaging without using grid. (b) Imaging with using grid

\subsection{Evaluation of Image Contrast}

The contrast of image is estimated by calculating the contrast index for each X-ray image which is the difference between the points of minimum and maximum optical density (OD) of steps wedge phantom. This procedure is in agreement with Meeson et al. (2014) work which calculated the contrast index in mammogram as the difference between the points of maximum and minimum OD in the main breast.

The OD of the film is read with a densitometer three times for each point in image to verify the consistency of results, the calculated measured doses are the average of those measurements. The uncertainty of measurement was estimated for all tests and the actual results are in the range of acceptable values range within $\pm 5 \%$.

The experiments in this study are conducted in the Biophysics laboratory, School of Physics, University of Science Malaysia (USM), Malaysia. 


\section{Results and Discussion}

\subsection{Image Contrast at Various X-ray Energy $(\mathrm{kVp}$ )}

Obviously, the X-ray image is formed when an X-ray photon with different densities collide with the film - screen combination in form of latent image which appears after adequate processing for X-ray film

The different intensities of incident photons on image's receptor determine the image contrast (optical density variation for adjacent components of radiograph). The optical density is determined by the amount of X-ray photons which interact with image receptor, hence, scattering radiation can affect the radiographic image contrast and the density (viewing the overall general image darkness or lightness). The reduction of backscattered radiation (occurred by using anti-backscattered grids under film screen during exposure)on the image contrast is studied different exposure parameters $(50 \mathrm{kVp}-110 \mathrm{kVp})$. A range of incident X-ray energies are utilized to obtain radiographic image with and without using fabricated grids. The contrast indices for all setups are obtained with exposure intensity is fixed at $20 \mathrm{mAs}$. Whereas, the distance between X-ray tube's focal spot and the image detector (FDD) is adjusted at $100 \mathrm{~cm}$.

Figure 4 illustrates the contrast indices as a function of the X-rays energy $(\mathrm{kVp})$ for different images with and without using anti-backscattered grids.

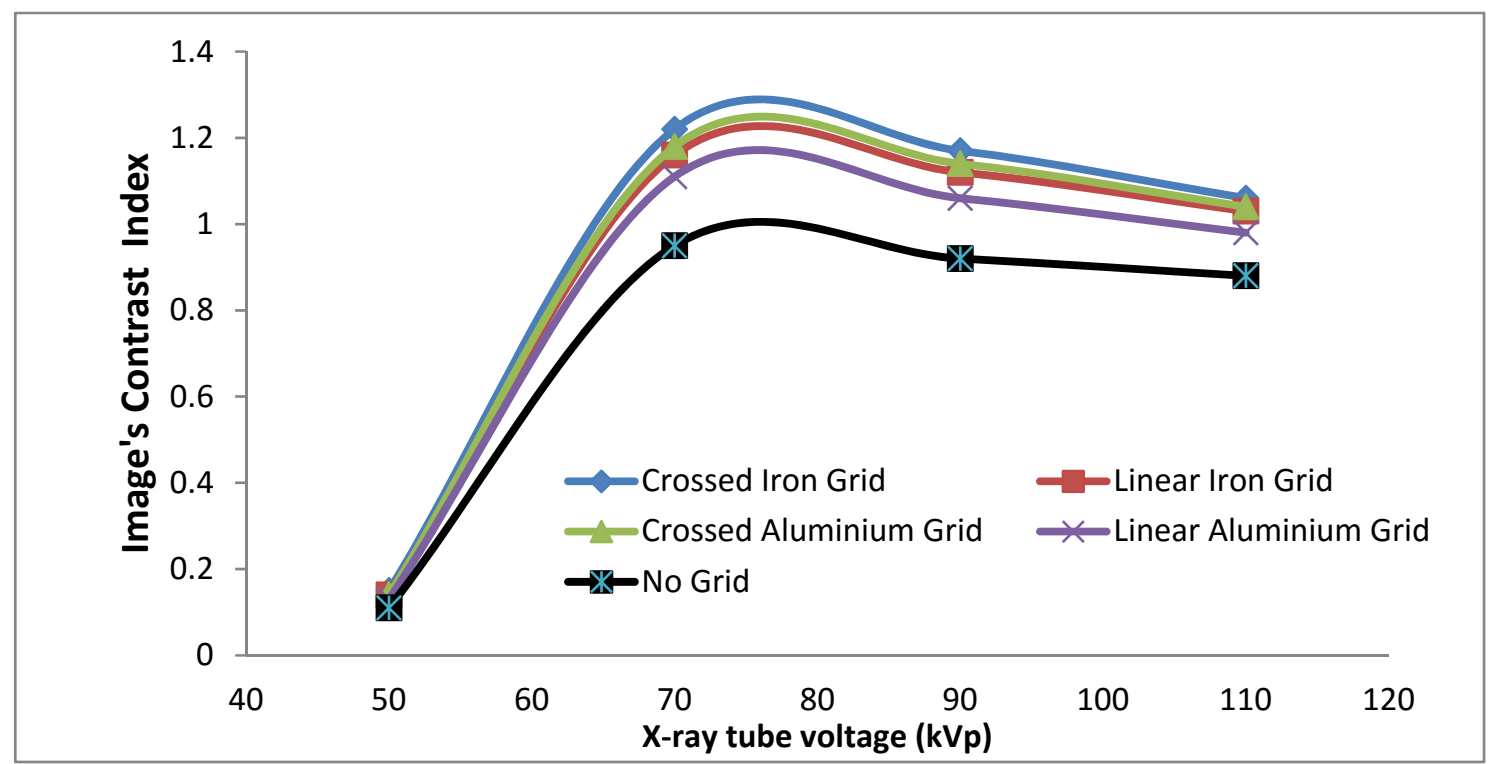

Figure 4. Image contrast indices at different $\mathrm{X}$-ray tube voltages

Image contrast depends on some parameters including potential voltage of the X-ray tube and presence of scattered radiation. The choice of peak tube voltage $(\mathrm{kVp})$ plays an important role in the radiographic image contrast as well as in the radiograph density.

Primary photons energy should not be too weak because much loss in radiographic image's contrast occurs with lower energy incident photons $(50 \mathrm{kVp}$ ). For lower range of X-rays energy (less than $70 \mathrm{kVp}$ ), the contrast increased by increasing photons energy because more photons can penetrate aluminium steps wedge and expose film, consequently, the variation for attenuation levels and optical densities can be obtained.

With reference to optimum contrast, figure 4 shows that the best contrast index for aluminium steps wedge can be obtained with tube peak voltage of $70 \mathrm{kVp}$, as the relatively moderate energies more adequate to image aluminium steps wedge with better contrast.

In contrast, by increasing the X-ray photons energy (more than $70 \mathrm{kVp}$ ), the higher voltages provide more penetrability for x-ray photons but less image contrast. Furthermore, the contrast indices are decreased by increasing tube's voltage for all setups because the more scatter radiation reaches to the image receptor, the more noise for radiograph will be. Consistent results are achieved by other authors (Huda \& Abrahams, 2015; Hendee \& Ritenour, 2003; Hobbie \& Roth, 2007; Fletcher \& McCollough, 2010; Alsleem, 2014) who reported that low 
energy is usually associated with higher contrast and vice versa. Suetens (2009) also reported that the higher the contrast is, the lower the useful exposure range is.

Using fabricated anti-backscattered grids improve the contrast indices at various $\mathrm{kVp}$, according to grid's effectiveness in reducing backscattered radiation. This result is in agreement with Hobbie and Roth (2007) who reported that similar Compton-scattered interactions, which collides the film, reduces the contrast, and contributes in the overall background darkening. These interaction scan be reduced by using an anti-scatter grid. In addition, backscattered photons produce unwanted exposure density without acquiring any pertinent information to the image receptor (film screen combination).

The energy of primary photons of X-ray plays an essential role in the improvement of image contrast index. To compare the effectiveness of different fabricated grids at different X-rays energy $(\mathrm{kVp})$, the percentages of increase in image's contrast indices - by utilizing several grids- are calculated at different X-rays tube voltage ranged from $50 \mathrm{kVp}$ to $110 \mathrm{kVp}$. The percentage of contrast increasing is calculated by dividing the increase of contrast index as a result of the use of grid by the contrast index without grid. The percentage of contrast increasing can be used to evaluate the effectiveness of anti-backscattered grids in reducing backscattered radiation. Figure 5 illustrates the effect of using anti-backscattered grids on radiographic image contrast at different $\mathrm{kVp}$.

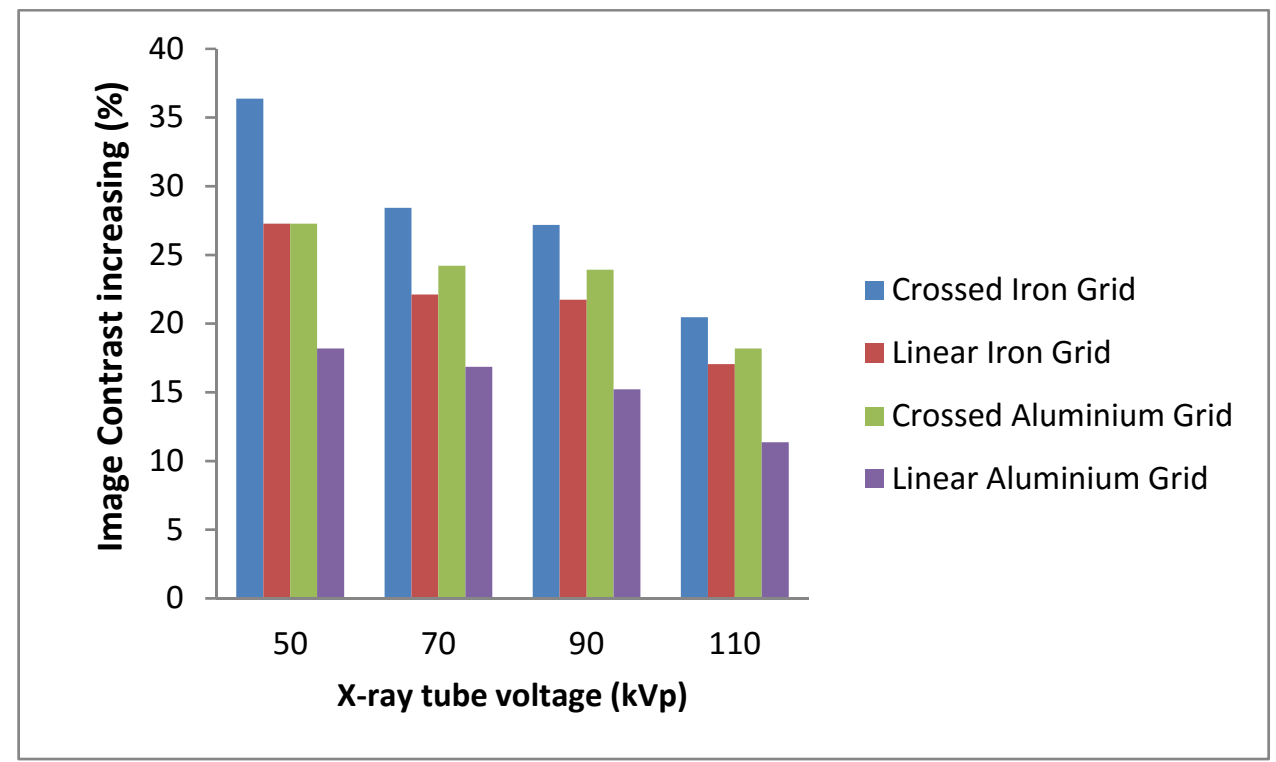

Figure 5. Increase of contrast indices by using anti-backscattered grids

Figure 5 indicates that the use of different grids proves a considerable increase for the radiographic image contrast at various applied voltages $(\mathrm{kVp})$. The increase of images contrast indices are related tothe reduction of back scattered radiation which in turn depends on the energy of remnant X-ray photons. Therefore, the best increment in contrast indexes, as a result of utilizing anti-backscattered grid, could be obtained by using lower X-rays energy $(\mathrm{kVp})$ due to the capability of grid to attenuate more backscattered photons with low energy. Different antibackscattered grids show the best values in enhancement of image contrast index at lower peak tube voltage $(50$ $\mathrm{kVp}$ ) because more backscattered photons can be attenuated by anti-backscattered grid by grid's base and strips. This result is in agreement with Naji et al. (2016) who report that the best reduction of backscattered radiation can be obtained with lower exposure's energy.

The increase of radiographic image contrast depends on the effectiveness of anti-backscattered grids. Therefore, by using several types of anti-backscattered grids namely; crossed iron, linear iron, crossed aluminium, and linear aluminium. The percentages of increasing in contrast indices are up to $36.363 \%, 27.272 \%, 27.272 \%$, and $18.181 \%$ respectively. As a result of the high correlation between image's contrast enhancement and backscattered radiation reduction.

The percentage of increase in image contrast index is decreased by the increasing of peak tube voltage $(\mathrm{kVp})$. The lowest percentages of increment to image contrast indices are obtained at high X-ray tube voltage $(110 \mathrm{kVp})$ because of the effect of primary photons energy on scattering interactions and backscatter photons quantity. 
Relevant to this study, Hendee and Ritenour (2003) reported that less contrast provided by a grid is obtained for an X-ray beam generated at a higher $\mathrm{kVp}$. In addition, Yaffe (2003) reports that scattered radiation recorded by the image receptor has the effect of creating a quasi-uniform haze on the image and causes the subject contrast to be reduced.

These results prove that the image's contrast index depend on the reduction of backscattered radiation and revealed the possibilities of improving image's contrast by using anti-backscattered grids.

In addition, the type of anti-backscattered grid plays an important role in the improvement of image contrast. To compare the effectiveness of different fabricated grids (crossed iron, linear iron, crossed aluminium and linear aluminium grid) in the improvement of radiographic image's contrast, the percentages of increase in image's contrast index (as a result of using anti-backscattered grids) are calculated at optimum X-rays energy for aluminium steps wedge imaging with best contrast $(70 \mathrm{kVp})$. Figure 6 illustrates the effectiveness of several fabricated anti-backscattered grids on the enhancement of radiograph contrast.

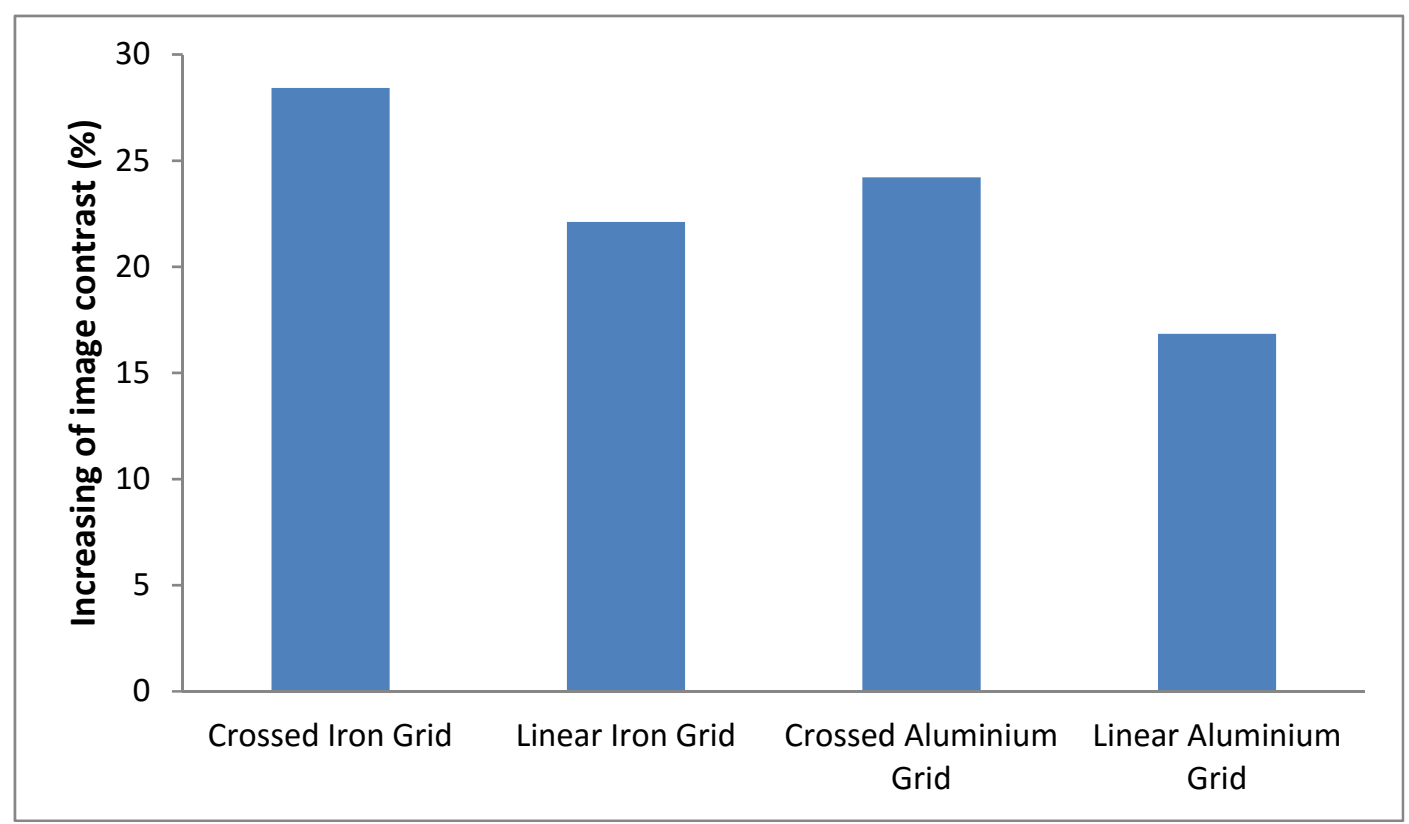

Figure 6. Increase of contrast indices by using different anti-backscattered grids

The increase of image contrast indices depends on the capability of anti-backscattered grid in attenuating backscattered radiation which in turn depends on the grid's material and geometrical design. Hence, the best enhancement in contrast indices is achieved by using crossed iron grid, because of the grid's geometrical design which trap more backscattered photons as well as the atomic number and density of grid's material which in turn enhance the effectiveness of grid.

At optimum X-rays energy to image aluminium step wedge $(70 \mathrm{kVp})$, the percentages of increase in contrast index up to about $28.42 \%, 22.11 \%, 24.21 \%$ and $16.84 \%$. These percentages obtained by using anti-backscattered grids type of crossed iron, linear iron, crossed aluminium, and linear aluminium, respectively. The results of image's contrast indicate that an efficient way to enhance radiographic image contrast can be achieved by using antibackscattered grid.

\subsection{Image Contrast Variation at Various Mas}

The exposure intensity (mAs) is one of the affecting factors on image contrast. This section evaluates the effect of exposure intensity (mAs) on the image's contrast as well as the effectiveness of anti-backscattered grids at different exposure intensity. Therefore, different mAs are used to image contrast test tool (aluminium steps wedge) with / out using anti-backscattered grids. All exposures are obtained at fixed X-rays energy for optimum contrast (70 $\mathrm{kVp}$ ), the effect of mAs on the image contrast with different exposure intensities is demonstrated in Figure 7. 


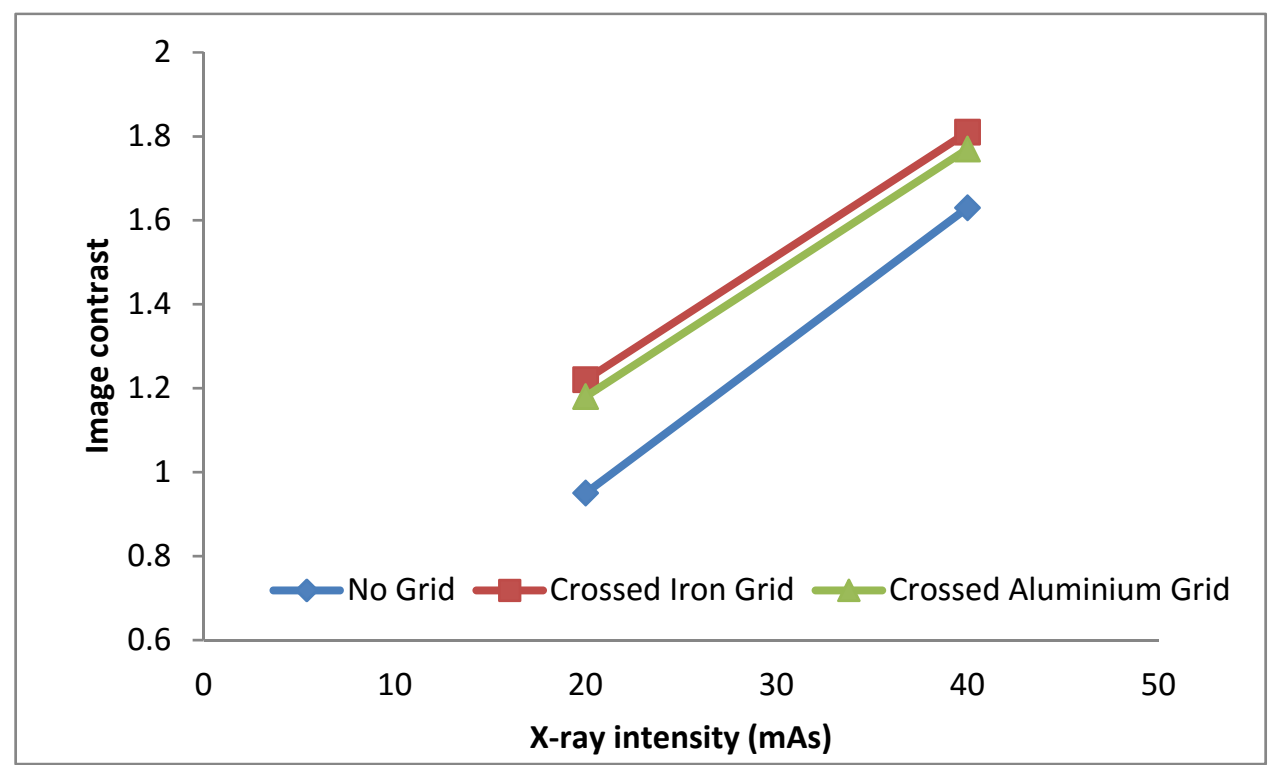

Figure 7. Image's contrast indices at different mAs

Figure 7 demonstrates that the effect of exposure intensity (mAs) on image contrast depends on the statistical nature (quantity) of incident and remnant photons as well as scattered ratio of X-ray photons. Image contrast indices increase by increasing mAs as a result of the nature of imaged object (Aluminium steps wedge) in attenuating X-ray photons (subject contrast) which caused more variation in optical densities for adjusted areas of $\mathrm{X}$-ray image. The increase of exposure intensity provides more variation in optical density of aluminium steps wedge because more $\mathrm{X}$-ray photons can reach radiographic film. Therefore, the image contrast indices present the best values with higher exposure intensity $(40 \mathrm{mAs})$.

This result is inconsistent with that of Thompson et al. (2015) who suggest that the optimized lesion detection in chest phantom model can be obtained at 40 mAs. Similarly, Sun et al. (2015) report that the reduction of mAs for cone-beam CT scans will lead to degradation of projection images, where more photons give less noise and fluctuation on the optical density.

In summary, the contrast index increases by the increase of optical densities variation between two adjacent areas of radiograph as a result of using anti-backscattered grids. The improvement in contrast index depends on the grid ability in reducing backscattering radiation which is randomly distributed through the film's surface area and thus reduce optical densities variation. To estimate the effectiveness of different anti-backscattered grids on the enhancement of image contrast at different mAs, the increasing percentages of contrast index, as a result of using different anti-backscattered grid are calculated from the results of contrast index with and without using grids as illustrated in Figure 8.

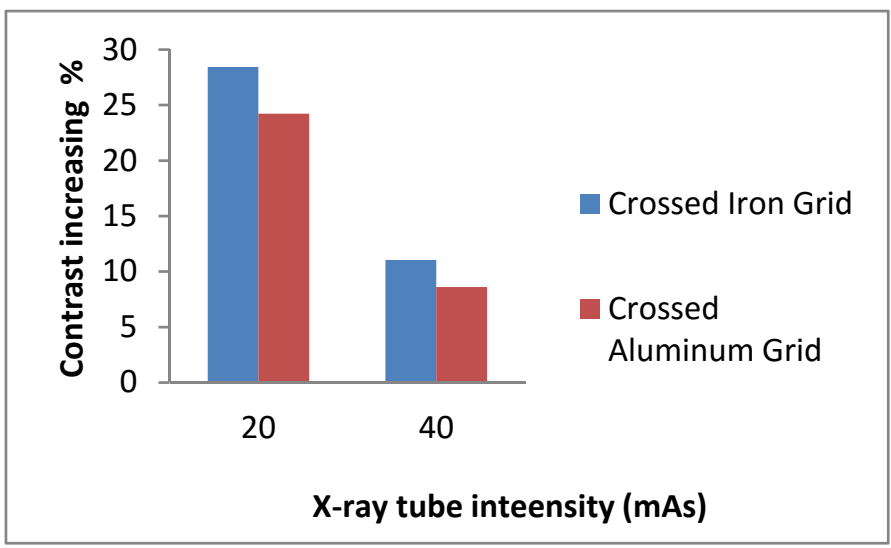

Figure 8. Contrast increasing percentage at different $\mathrm{mAs}$ 
The percentages of contrast increasing by using anti-backscattered grids are better with lowerX-ray tube current $(20 \mathrm{mAs})$. These results can be attributed to the statistical nature of measurements, because of the ratio of backscattered reduction that is resulted by utilizing grids is higher at lower exposure intensity ( $20 \mathrm{mAs})$. The increasing percentage in image contrast depends on the reduction of backscattered photons.

With reference to anti-backscattered grid type, figure10shows that at both $\mathrm{mAs}$ the cross iron grid provide higher impact in improvement of image contrast index compared with crossed aluminium grid. This is because grid's material (iron and/or steel) which has better capability in attenuating of backscattered radiation. This result is in agreement with Naji et al. (2016) who reported that the iron steel grid has best value in reducing backscattered radiation during $\mathrm{X}$-ray examinations

\subsection{The contribution of this study can be summarized as follows:}

Imaging system with film screen combination require the enhancement of image contrast in order to provide good quality particularly in area limited by advanced diagnostic systems such as digital radiography. For instance, conventional radiography based on film cassette combination accounts for $88 \%$ of $\mathrm{x}$-ray-based imaging in Yemen, the conventional radiography is responsible for the most of the radiation dose associated with all medical imaging techniques (NAEC, 2015).

The approach developed in this study is directly applicable to any measurement with minimal complexity. This study showed that it is possible to increase the contrast of images up to $36 \%$ with the use of anti-scatter crossed iron grids placed under film screen combination during exposure. Therefore, the optimization of backscattered dose and image quality relationship outlined in this study are feasible and effective in clinical practice. Conclusively, the results of this study can be applied to develop guidelines for the reduction of backscattered radiation using anti-scatter grids.

\section{Conclusion}

The X-ray image contrast depends on various factors such as exposure parameters, subject contrast and backscattered radiation existence. Image contrast can be evaluated by assessment of optical density variation between two adjacent areas on radiograph (using aluminium steps wedge). Radiographic image contrast can be enhanced by controlling the backscattered radiation, as well as the exposure parameters. Moreover, all anti-back scattered grids fabricated in this work prove remarkable capability in reducing back scattered radiation and improving image's contrast. The effectiveness of fabricated grids in reducing backscattered radiation is dependent on the grid's material and design, as well as the radiation exposure parameters. Therefore, better X-ray image contrast can be obtained by using anti-backscattered crossed grids fabricated from iron steel with exposure parameters $(70 \mathrm{kVp}, 40 \mathrm{mAs})$. Whereas the percentage of image contrast increasing up to $36 \%$. These results prove applicable method to reduce backscattered radiation and improve X-ray image quality.

\section{References}

Alsleem, H. A. (2014). Evaluation of factors that affect contrast-detail in digital X-Ray and computed tomography. $\mathrm{PhD}$ thesis, RMIT University, Australia.

Bushberg, J. T., \& Boone, J. M. (2012). The essential physics of medical imaging (3rd ed.). Lippincott Williams \& Wilkins, USA.

Bushong, S. C. (2013). Radiologic science for technologists: physics, biology, and protection (10th ed.). Elsevier Health Sciences.

Carver, E., Carver, B., Elizabeth, B., \& Price, R. C. (2012). Medical imaging: techniques, reflection and evaluation (2nd ed.). Elsevier Health Sciences.

Fletcher, C. (2010). Adjusting kV to Reduce Dose or Improve Image Quality - How to Do it Right. Medical Physics, 37(1). Mayo CT Clinic Innovation Center and Dept. of Radiology: Technology Assessment Institute: Summit on CT Dose. Retrieved from https://www.aapm.org/meetings/2010CTS/documents/1130Fletcher-AutokV04-29-2010.pdf.

Godwin, D., Edward, S. P., Krishna, K. S., Kishan, K. K., Chitra, P. \& Nandhitha, N. M. (2014). Survey on Contrast Enhancement Techniques for Medical X-Ray Images to Highlight The Abnormalities. Int. Journal of Engineering Research and Applications, 4(4), 19-24.

Guy, C., \& Flytche, D. (2005). An introduction to the principles of medical imaging. Imperial Coll. Press. USA.

Hendee, W. R., \& Ritenour, E. R. (2003). Medical imaging physics. John Wiley \& Sons, New York. USA. 
Hobbie, R., \& Roth, B. J. (2007). Intermediate physics for medicine and biology (4th ed.). Springer Science \& Business Media, New York, USA.

Huda, W., \& Abrahams, R. B. (2015). Radiographic techniques, contrast, and noise in x-ray imaging. American Journal of Roentgenology, 204, 126-131.

Meeson, S., Young, K., Ramsdale, M., Wallis, M., \& Cooke, J. (2014). Analysis of optical density and contrast in mammograms. The British Journal of Radiology, 72, 670-677.

Mohanta, K., \& Khanaa, V. (2013). An Efficient Contrast Enhancement of Medical X-Ray Images-Adaptive Region Growing Approach. International Journal of Engineering and Computer Science, 2, 208-212.

NAEC, National Atomic Energy Commission. (2015). Statistics of Ionizing Radiation Sources Used in Medical Diagnostic in Yemen. technical report, Ministry of Health, Sana'a, Yemen.

Naji, A. T. (2016). Fabrication of anti-scatter radiation for reduction of backscattered radiation and evaluate its effectiveness on x-ray image quality. PhD Thesis, University of Science Malaysia. USM.

Naji, A. T., Jaafar, M. S., Ali, E. A., \& Al-Ani, S. K. J. (2016). X-ray Attenuation and Reduction of Backscattered Radiation. Applied Physics Research, 8(4), 92-102. http://dx.doi.org/10.5539/apr.v8n4p92

Suetens, P. (2009). Fundamentals of medical imaging (2nd ed.). Cambridge university press, The Edinburgh Building, UK.

Sun, N., Sun, T., Wang, J., \& Tan, S. (2015). CBCT reconstruction via a penalty combining total variation and its higher-degree term. In: SPIE Medical Imaging. International Society for Optics and Photonics, 94123-94129.

Thompson, J. D., Chakraborty, D. P., Szczepura, K., Vamvakas, I., Tootell, A., Manning, D. J. \& Hogg, P. (2015). A phantom-based JAFROC observer study of two CT reconstruction methods: the search for optimisation of lesion detection and effective dose. In SPIE Medical Imaging, International Society for Optics and Photonics, 94160-94166.

Yaffe, M. J. (2003). Mammography, Biomedical Imaging, The Biomedical Engineering Handbook (Vol. I, 2nd ed.). Joseph D. Bronzino, CRC Press, Boca Raton, FL, USA.

Yumpu. (2015). Physics of Medical X-Ray Imaging. Retrieved May 12th, 2015, from https://www.yumpu.com/en/ document/view/11544742/ chapter-4-physical-determinants-of-contrast.

\section{Copyrights}

Copyright for this article is retained by the author(s), with first publication rights granted to the journal.

This is an open-access article distributed under the terms and conditions of the Creative Commons Attribution license (http://creativecommons.org/licenses/by/4.0/). 